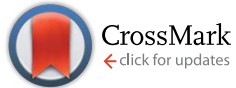

Cite this: Chem. Sci., 2015, 6, 1887

Received 23rd October 2014 Accepted 22nd December 2014 DOI: $10.1039 / c 4 s c 03258 f$

www.rsc.org/chemicalscience

\section{Principal factors that determine the extension of detection range in molecular beacon aptamer/ conjugated polyelectrolyte bioassays $\dagger$}

\author{
Ji-Eun Jeong, ${ }^{a}$ Boram Kim, ${ }^{a}$ Shinjae Woo, ${ }^{a}$ Sungu Hwang, ${ }^{\text {b }}$ Guillermo C. Bazan*c \\ and Han Young Woo*a
}

A strategy to extend the detection range of weakly-binding targets is reported that takes advantage of fluorescence resonance energy transfer (FRET)-based bioassays based on molecular beacon aptamers (MBAs) and cationic conjugated polyelectrolytes (CPEs). In comparison to other aptamer-target pairs, the aptamer-based adenosine triphosphate (ATP) detection assays are limited by the relatively weak binding between the two partners. In response, a series of MBAs were designed that have different stem stabilities while keeping the constant ATP-specific aptamer sequence in the loop part. The MBAs are labeled with a fluorophore and a quencher at both termini. In the absence of ATP, the hairpin MBAs can be opened by CPEs via a combination of electrostatic and hydrophobic interactions, showing a FRETsensitized fluorophore signal. In the presence of ATP, the aptamer forms a G-quadruplex and the FRET signal decreases due to tighter contact between the fluorophore and quencher in the ATP/MBA/CPE triplex structure. The FRET-sensitized signal is inversely proportional to [ATP]. The extension of the detection range is determined by the competition between opening of the ATP/MBA G-quadruplex by CPEs and the composite influence by ATP/aptamer binding and the stem interactions. With increasing stem stability, the weak binding of ATP and its aptamer is successfully compensated to show the resistance to disruption by CPEs, resulting in a substantially broadened detection range (from millimolar up to nanomolar concentrations) and a remarkably improved limit of detection. From a general perspective, this strategy has the potential to be extended to other chemical- and biological-assays with low target binding affinity.

\section{Introduction}

Conjugated polyelectrolytes (CPEs) have emerged as attractive and versatile optical platforms for highly sensitive chemo- or biosensors ${ }^{1-5}$ that can detect a variety of targets, including metal ions, DNA, RNA and peptides. ${ }^{6-11}$ CPEs are described by a $\pi$ conjugated backbone with ionic pendant groups, which make them water-soluble and/or bio-compatible. A range of cationic and anionic CPEs have been synthesized for specific applications including bioimaging, bioelectronics, fluorescent probes and signaling mediation. ${ }^{\mathbf{1 2 - 1 7}}$ Structural diversity allows the optical and electronic properties of CPEs to be adjustable

\footnotetext{
${ }^{a}$ Department of Nanofusion Engineering, Department of Cogno-Mechatronics Engineering, Pusan National University, Miryang, Gyeongsangnam-do 627-706, Republic of Korea. E-mail: hywoo@pusan.ac.kr

${ }^{b}$ Department of Nanomechatronics Engineering, Pusan National University, Miryang, Gyeongsangnam-do 627-706, Republic of Korea

${ }^{c}$ Department of Chemistry \& Biochemistry, University of California, Santa Barbara, California 93106, USA. E-mail: bazan@chem.ucsb.edu

$\dagger$ Electronic supplementary information (ESI) available: Additional UV-vis, PL and CV data, equilibrium constant calculation for MBAs conformational change, and CD data. See DOI: 10.1039/c4sc03258f
} properties..$^{18,19}$

Molecular beacons (MB) are loop-stem hairpin-type signaling probes labeled with a fluorophore and a quencher at the two different termini of the stem. This structural feature in the hairpin structure leads to a substantially decreased photoluminescence (PL) emission by virtue of the fluorophore and quencher close proximity. In the presence of a complementary target, the MB probe undergoes a conformational change from hairpin to an open-chain structure, resulting in the recovery of PL from the fluorophore. Interesting strategies were recently reported to control the detection range of MB-based DNA assays by modulation of stem stability. For example, it has been reported that the stem length has a significant impact on the binding characteristics and hybridization kinetics of MBs. With increasing the stem length, target discrimination can be facilitated over a broader range of temperature but a decrease in the rate of $\mathrm{MB} /$ target hybridization was measured. ${ }^{20}$ Another approach for tuning the detection range has been demonstrated by controlling the stem stability of MB probes. Specifically, the detection range can be shifted toward higher target concentration with increases in the stability of the nonbinding hairpin and consequently takes advantage of their light harvesting 
structure. ${ }^{21,22}$ In an another electrochemical bioassay where a redox reporter-modified $\mathrm{MB}$ was attached to the electrode, hybridization of MB with a complementary target DNA induced stretching of the hairpin structure away from the electrode, giving rise to decreasing electron transfer from the reporter to electrode. This process was successfully modulated by changing the MB stem stability. ${ }^{23}$

We recently demonstrated a highly sensitive and selective potassium $\left(\mathrm{K}^{+}\right)$detection assay with a limit of detection (LOD) of $\sim 1.5 \mathrm{nM}$ based on a cationic poly(fluorene-co-phenylene) based CPE (PPFP-Br) and a oligonucleotide molecular beacon aptamer (MBA) containing a $\mathrm{K}^{+}$-specific aptamer base sequence in its loop part (Scheme 1). ${ }^{24}$ Aptamers are well-recognized targetspecific biomolecules that may serve as recognition elements in biological assays, including those mediated by CPEs. ${ }^{25-27}$ They are defined as nucleic acid based ligands with high binding affinities toward broad range of molecules. ${ }^{28,29}$ In particular, single-stranded DNA aptamers with guanine (G)-rich sequences can bind to a given target and fold into a secondary G-quadruplex structure via intramolecular hydrogen-bonding interactions. ${ }^{30-32}$ The MBA is an aptamer-based MB containing a specific aptamer base sequence in the loop structure. Structural reorganization from hairpin to an open-chain conformation is induced by complexation with cationic CPEs. ${ }^{33,34}$ The open MBA structure in the presence of CPE shows a strong fluorescence resonance energy transfer (FRET)-induced fluorophore emission in the absence of $\mathrm{K}^{+}$ions (on state). Upon addition of $\mathrm{K}^{+}$ ions, the MBAs prefer the G-quadruplex conformation, even in the presence of cationic CPEs, which leads to a decrease in PL (off state). Monitoring the CPE-sensitized fluorophore reporter emission intensity thus allows one to detect $\mathrm{K}^{+}$. This MBA/CPE based detection approach is operationally simple and may be extended to a wide range of target materials by modifying the aptamer sequence in the loop.

Based on the above studies, we describe in this contribution a novel strategy that combines biomolecular MBAs and a synthetic polymer, to tune the detection range for weakly binding target materials. We also provide the principal factors that govern the detection mechanisms. ATP was selected as the
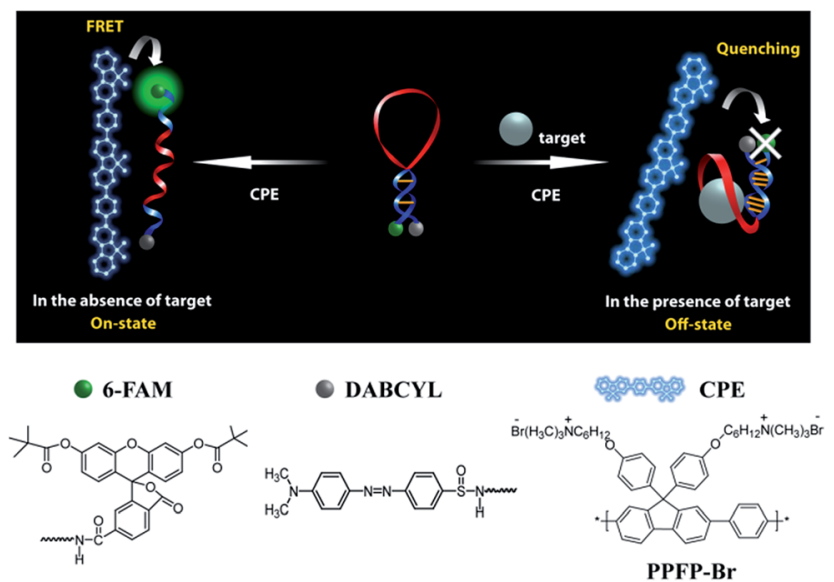

Scheme 1 Schematic for MBA/CPE-based bioassay. target material due to the relatively weak binding constant with its complementary aptamer. Specifically, a dissociation constant $\left(K_{\mathrm{d}}\right)$ of $\mu \mathrm{M}-\mathrm{mM}$ was reported previously for the ATP/ aptamer complex. ${ }^{35-39}$ For comparison, a $K_{\mathrm{d}}$ value in the range of 2.68-450 $\mathrm{nM}$ was reported for $\mathrm{K}^{+} /$aptamer binding. ${ }^{40}$ Doxorubicin and human VEGF (vascular endothelial growth factor) can be captured by its aptamer with $K_{\mathrm{d}}=600 \mathrm{nM}$ and $50 \mathrm{pM}$, respectively, ${ }^{\mathbf{4 1 , 4 2}}$ and the lysozyme aptamer binds to its target with $K_{\mathrm{d}}=31 \mathrm{nM}^{43}$

A series of MBAs (MBA1-MBA5) were designed so that the differences in structure lead to different stem stabilities. The MBAs contain the same ATP-specific aptamer sequence within the loop substructure, while their stem interactions were finetuned by changing the number of guanine (G)-cytosine (C) triple hydrogen bonds. As described in detail, the extension of the detection range depends critically on the competition between opening of the ATP/MBA G-quadruplex by CPEs and the composite influence by ATP/aptamer binding and the stem interactions. With increasing stem stability (MBA1 $\rightarrow$ MBA5), the weak binding affinity of ATP and its aptamer was successfully compensated to show resistance to opening by CPEs, resulting in a significantly broadened detection range with remarkably improved LOD: from millimolar up to nanomolar concentrations.

\section{Results and discussions}

\section{MBA/CPE-based detection scheme}

Scheme 2 provides a sequence of relevant intramolecular conformational changes and intermolecular processes that form the basis of the MBA/CPE based FRET assay for ATP detection. The MBA structure contains a fluorophore (6-carboxyfluorescein (6-FAM), F) and a quencher (4-(4'-dimethylaminophenylazo)benzoic acid (DABCYL), Q) at the two different termini. At room temperature equilibrium, MBA exists primarily as a hairpin-type structure, MBA-closed (step 1, $K_{1}$ ). The aptamer sequence of $5^{\prime}$ GGGG AGTA TTGC GGAG GA $3^{\prime}$, which has been reported to form a G-quadruplex specifically upon interaction with ATP, ${ }^{35,44}$ is incorporated into the loop substructure of MBA. Treatment of the MBA with ATP leads to a mixture of open-chain MBA, hairpin MBA, unbound ATP and the ATP/MBA complex (see step 2). The exact relative population of these species will be determined by the magnitude of the equilibrium constants $K_{1}$ and $K_{2} . K_{1}$ becomes larger with increasing the hydrogen bonding interactions in the MBA stem substructure. $K_{2}$ is determined by the ATP-aptamer interaction, which can be made nearly constant for all MBAs by using the same oligonucleotide sequence. Consider now step 3. Four types of electrostatic complexes may form immediately after CPE addition, including: open-chain MBA/CPE (1), hairpin MBA/CPE (2), a triplex of ATP/MBA/CPE (3) and ATP/CPE. However, a final equilibrium state will develop over time through opening of the hairpin structures by CPE. For instance, disruption of species 2 generates open-chain MBA/CPE, which in Scheme 2 is illustrated as $1^{\prime}$ to aid in subsequent discussion. Similarly, opening of species 3 yields $1^{\prime \prime}$. These two processes are contained in the red box of step 3. Greater FRET PL intensity 


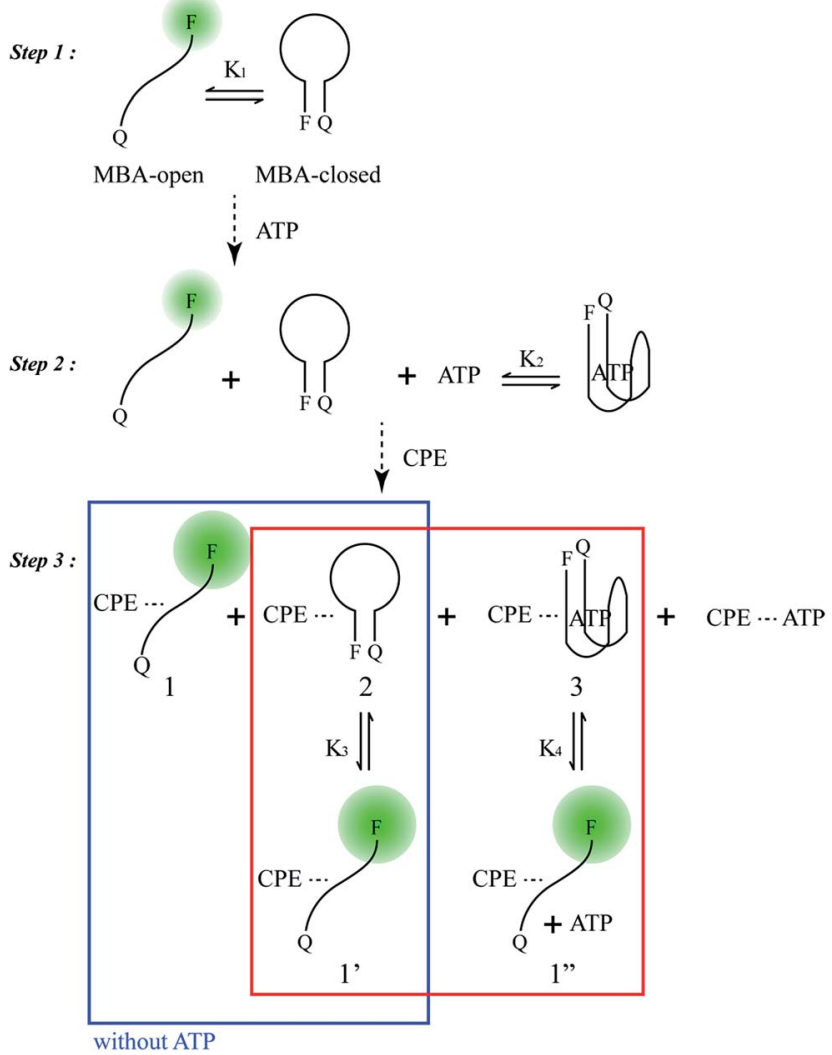

Scheme 2 Principal participants in the ATP detection mechanism in the MBA/CPE assay.

and FRET ratio due to the higher population of open-chain $\mathrm{MBA} / \mathrm{CPE}$ would be expected in the absence of ATP (blue box in step 3). After treatment with ATP, the resulting FRET signal comes from the open-chain MBA/CPE complex, i.e. the sum total of species, $1,1^{\prime}$ and $1^{\prime \prime}$, in step 3 , where the concentration of 1 is negligible with increasing stem stability and the total concentration of the emissive open-chain $\mathrm{MBA} / \mathrm{CPE}$ is inversely proportional to [ATP].

A series of experiments were carried out to validate Scheme 2. First, we examined the general basis of ATP/MBA/CPE interactions for providing a response toward [ATP]. We focused on MBA1 and the CPE, PPFP-Br. The molecular structure of MBA1 can be found in Fig. 2. Fig. 1 shows the normalized FRET PL
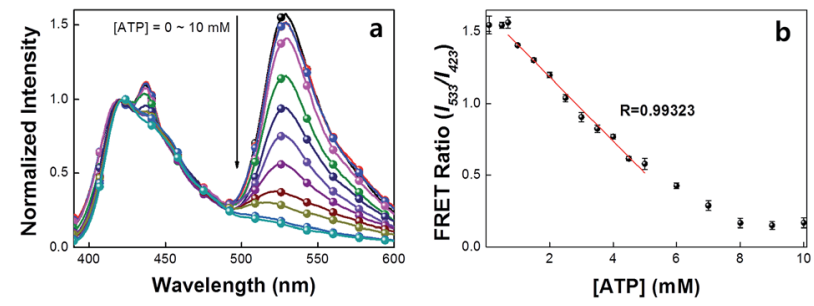

Fig. 1 (a) Normalized FRET PL spectra and (b) detection range of MBA1/PPFP-Br. FRET spectra were obtained by exciting at $380 \mathrm{~nm}$. $[\mathrm{PPFP}-\mathrm{Br}]=1.0 \times 10^{-6} \mathrm{M},[\mathrm{MBA} 1]=2.0 \times 10^{-8} \mathrm{M}$. The FRET ratio shows linearity in the range of $\sim \mathrm{mM}$ ATP concentration range. spectra (upon excitation of PPFP-Br at $380 \mathrm{~nm}$ ) of MBA1/PPFP$\mathbf{B r}$ with changing $[\mathrm{ATP}]$ in Tris-HCl buffer $([\mathbf{P P F P}-\mathrm{Br}]=1.0 \times$ $\left.10^{-6} \mathrm{M},[\mathrm{MBA1}]=2.0 \times 10^{-8} \mathrm{M}\right)$. One observes from Fig. 1 that the FRET-sensitized 6-FAM signal and the FRET ratio $\left(I_{533} / I_{423}\right.$, where $I_{533}$ is the PL intensity at $533 \mathrm{~nm}$ and $I_{423}$ is the intensity at $423 \mathrm{~nm}$ ) gradually decrease with increasing [ATP]. Under these conditions the ATP/MBA1 G-quadruplex concentration, namely species 3 in Scheme 2, increases and the resulting FRETinduced 6-FAM signal decreases. The FRET ratio shows a linear relationship in a range of $[\mathrm{ATP}]=1.0 \times 10^{-3} \mathrm{M}$ to $7.1 \times 10^{-3} \mathrm{M}$, from which a quantitative ATP detection calibration curve can be constructed for the MBA1/PPFP-Br combination, as shown in Fig. $1 \mathrm{~b}$. The detection range was determined by fitting a titration curve using the Hill equation, where the FRET ratio transits from $10 \%$ to $90 \%$ of its signal output. ${ }^{45,46}$ The LOD ( $3 \sigma /$ slope, $\sigma$ is the standard deviation from 4 independent measurements of the slope of the plot in Fig. 1b) was determined to be $5.7 \times 10^{-4}$ M for MBA1/PPFP-Br. The data in Fig. 1 therefore provide a preliminary demonstration that the MBA1/PPFP-Br combination provides a quantitative analysis of [ATP] via the sequence of steps in Scheme 2.

\section{Modulation of detection characteristics by changing stem stability in MBAs}

While the data in Fig. 1 demonstrate that the MBA1/PPFP-Br combination provides a successful detection of ATP, the detection sensitivity is limited (LOD $=0.57 \mathrm{mM}$ ) due to the weak binding $\left(K_{\mathrm{d}}=1 / K_{2}=\mu \mathrm{M}-\mathrm{mM}\right)$ between ATP and the aptamer sequence in MBA1. ${ }^{35-39}$ An efficient way to tune the sensitivity range by compensating the weak binding strength of ATP/aptamer is therefore required. Of particular practical relevance is that a limited detection range complicates, or in some instances precludes, the general use of biosensors in many real applications.

Fig. 2 shows the molecular structure of the five MBAs in our studies. They all contain the same ATP-specific aptamer

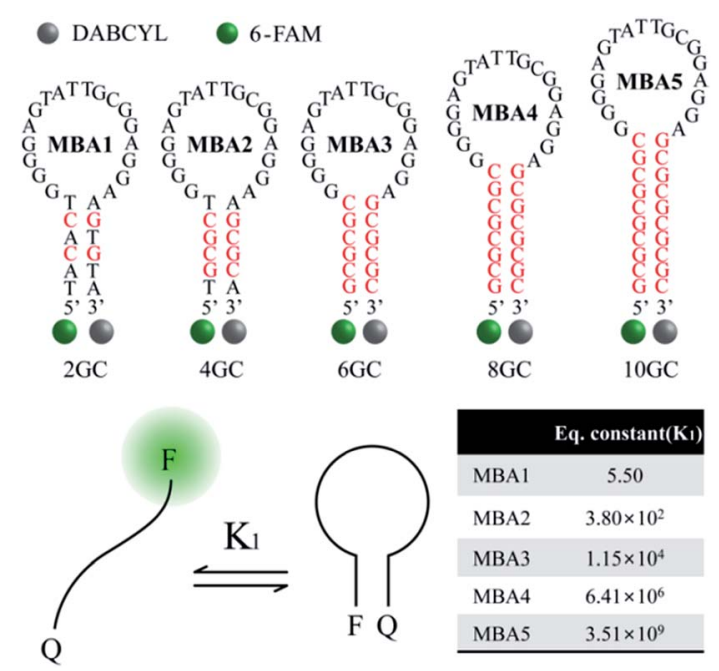

Fig. 2 Molecular structures of the five MBAs used in the studies, together with calculated $K_{1}$ values. 
sequence in the loop substructure but are anticipated to exhibit different stem stabilities due to the different number of $\mathrm{G}-\mathrm{C}$ base pairs in the stem. In Scheme $2, K_{2}$ is not anticipated to be greatly influenced by the stem stability. This same binding affinity for all MBAs was identified by a series of experiments described below. MBA1, 2, 3, 4 and 5 have two, four, six, eight and ten $\mathrm{G}-\mathrm{C}$ hydrogen bonds in the stem part, respectively. The equilibrium constant $\left(K_{1}\right.$, step 1$)$ for the conformational transformation between the open-chain and hairpin structures was calculated to be $K_{1}=5.5$ for MBA1, $3.8 \times 10^{2}$ for MBA2, $1.2 \times$ $10^{4}$ for MBA3, $6.4 \times 10^{6}$ for MBA4 and $3.5 \times 10^{9}$ for MBA5 (Table $\mathrm{S} 1 \dagger$ ). ${ }^{47,48}$ With increasing stem strength, the equilibrium is further shifted to the formation of the hairpin structure. Despite these different $K_{1}$ values, all MBAs show a decreased 6FAM emission by DABCYL when compared to analogous nonhairpin oligonucleotide sequences.

Prior to studying overall detection characteristics, the opening effect of the MBAs by PPFP-Br was investigated. These experiments relate to $K_{3}$ in Scheme 2. Fig. 3 compares the 6-FAM PL signals of MBA1-5 upon direct excitation of 6-FAM at 490 $\mathrm{nm}$, with and without the addition of PPFP-Br. With increasing [PPFP-Br] from 0 to $1.0 \times 10^{-6} \mathrm{M}$, the 6 -FAM signal gradually intensified. This increase is consistent with the opening of the hairpin structures by PPFP-Br. ${ }^{34}$ In the presence of $1 \mu \mathrm{M}$ PPFPBr, the 6-FAM signal intensity $(I)$ was enhanced by approximately 2.3, 2.1, 1.7, 1.2 and 1.1 times for MBA1, 2, 3, 4 and 5, respectively, relative to that without PPFP-Br $\left(I_{0}\right)$. As expected, the 6-FAM emission recovery by PPFP-Br decreased gradually with increasing stem stability, indicating the decreased
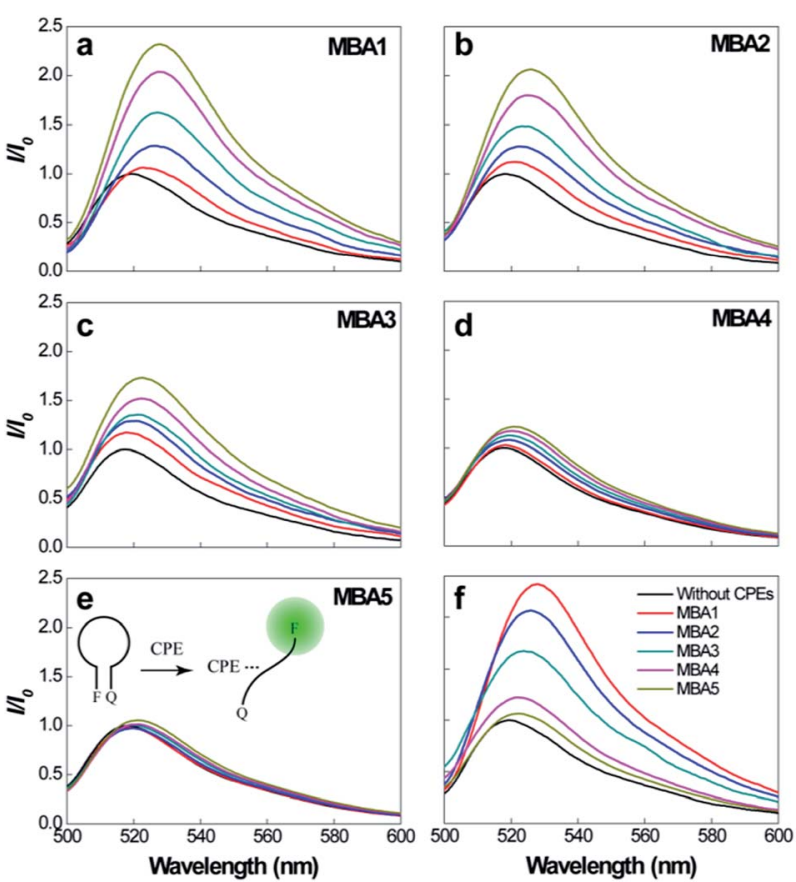

Fig. 3 (a)-(e) PL spectra of MBA1-5 (2.0 $\left.\times 10^{-8} \mathrm{M}\right)$ upon direct excitation of $6-F A M$ at $490 \mathrm{~nm}$, with increasing [PPFP-Br] $=0-1.0 \times$ $10^{-6} \mathrm{M}$. (f) Comparison of PL signals of different MBAs with [PPFP-Br] $=1.0 \times 10^{-6} \mathrm{M}$. capability to disrupt the hairpin structure upon electrostatic and hydrophobic interactions with PPFP-Br. Another perspective is that as $K_{1}$ increases, one obtains a decrease in $K_{3}$.

We now examine how the various MBA structures lead to differences in 6-FAM FRET sensitization. Fig. 4 shows the PL spectra of MBAs/PPFP-Br in the absence and presence of ATP by exciting PPFP-Br at $380 \mathrm{~nm}\left([\mathbf{P P F P}-\mathrm{Br}]=1.0 \times 10^{-6} \mathrm{M},[\mathrm{MBA}]=\right.$ $\left.2.0 \times 10^{-8} \mathrm{M}\right)$. Looking at the results with MBA1, a strong FRETinduced PL signal from 6-FAM was measured in the absence of ATP. This observation reflects the formation of an open-chain MBA1/PPFP-Br electrostatic complex and illustrates the onstate, where the quenching action by DABCYL is circumvented. The addition of excess ATP (50 mM) leads to quenching of the FRET-induced 6-FAM signal due to formation of the ATP/MBA1 G-quadruplex (off-state, species 3 in Scheme 2). A clear change in the emission color from greenish blue to dark blue of the MBA1/PPFP-Br solution can also be observed by naked eye upon ATP addition (Fig. 4, inset). Examination of the PL profiles also reveals that with increasing stem stability (MBA1 $\rightarrow$ MBA5), the FRET ratio exhibits a gradual decrease due to the decreased opening of MBAs by PPFP-Br without ATP. In the absence of ATP, the FRET-induced PL intensity depends on the sum of the concentrations of open-chain/CPE complexes derived from the sum of species 1 and $1^{\prime}$ (blue box in Scheme 2), which are impacted by the stem stability. From a biosensor design, it is relevant that the FRET signal and on/off ratio can therefore be simply modulated by modification of MBA stem stability.

The idea that all MBAs should have similar ATP binding capabilities is now examined. Fig. 5a shows the PL signals of MBA1 $\left(2.0 \times 10^{-8} \mathrm{M}\right)$ with changing $[\mathrm{ATP}]=0-9 \mathrm{mM}$ by exciting 6-FAM directly at $490 \mathrm{~nm}$ in the absence of PPFP-Br. The 6-FAM emission gradually decreases with increasing [ATP]. Such a decrease is consistent with further formation of the poorly emissive G-quadruplex structure possibly with a tightening of the internal structure such that the DABCYL quenching is accentuated. A plot of the intensity ratio $I / I_{0}$ (where $I$ is the 6FAM PL intensity at $517 \mathrm{~nm}$ and $I_{0}$ is the original intensity without ATP) against [ATP], as shown in Fig. 5b, provides a

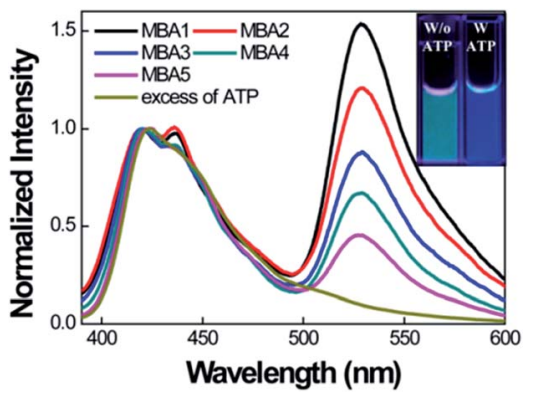

Fig. 4 Normalized FRET-induced PL spectra of MBAs/PPFP-Br by exciting at $380 \mathrm{~nm}$ in the absence of ATP. [PPFP-Br] $=1.0 \times 10^{-6} \mathrm{M}$, $[\mathrm{MBA}]=2.0 \times 10^{-8} \mathrm{M}$. The spectrum of MBA1/PPFP-Br with ATP $(50$ $\mathrm{mM}$ ) is displayed together for comparison. Inset shows the photograph of the solutions with (blue) and without (greenish blue) ATP under UV illumination at $365 \mathrm{~nm}$. Measurements were carried in $20 \mathrm{mM}$ Tris- $\mathrm{HCl}$ buffer, pH 7.4. 

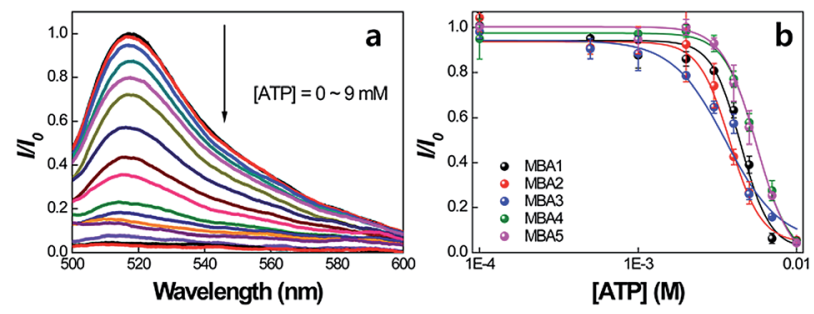

Fig. 5 (a) PL spectra of MBA1 with increasing [ATP] $=0-9 \mathrm{mM}$, and (b) $\mathrm{PL}$ intensity ratio $\left(/ / /_{0}\right)$ changes for MBAs as a function of [ATP]. $/$ is the 6-FAM PL intensity at $517 \mathrm{~nm}$ and $I_{0}$ is the original intensity without ATP. Emission spectra were obtained by exciting at $490 \mathrm{~nm}$. [MBAs] $=$ $2.0 \times 10^{-8} \mathrm{M}$.

spectral response profile. When this analysis is carried out for the five MBA structures one observes that they provide a similar response. Specifically, the five MBA probes show a similar millimolar concentration detection range, as determined by the ATP concentration regime through which $I / I_{0}$ transits from $10 \%$ to $90 \%$ of its signal output. These data allow us to estimate the dissociation constant, ${ }^{21} 1 / K_{2}$ to be on the order of $3.2-5.4 \mathrm{mM}$ and a LOD in the range of 1.8-2.7 $\mathrm{mM}$ for the five MBAs. The stem stability therefore does not appear to impact greatly the ATP/aptamer binding.

The impact of the interactions with PPFP-Br to extend the detection range is discussed next. Fig. 6 compares the FRET ratio of MBA/PPFP-Br assays with changing [ATP] from 0 to 50 $\mathrm{mM}$. These experiments were carried out by excitation of PPFPBr. In the case of MBA1 with the weakest stem, the FRET ratio, shown with the black trace, increases with decreasing [ATP], is fully recovered at $[\mathrm{ATP}]=\sim 5 \times 10^{-4} \mathrm{M}$, and demonstrates a linear spectral response within a $\sim \mathrm{mM}$ [ATP] range. Comparison of the plots for MBA1-4 shows that increasing the stem stability leads to a substantial extension of the spectral response range. These ranges can cover from millimolar $\left(1.0-7.1 \times 10^{-3}\right.$ M for MBA1/PPFP-Br), to micromolar $\left(7.2 \times 10^{-6}-7.0 \times 10^{-3} \mathrm{M}\right.$ for MBA2/PPFP-Br $)$ and ultimately nanomolar $\left(1.5 \times 10^{-8}-7.3 \times\right.$ $10^{-3} \mathrm{M}$ for MBA3/PPFP-Br and $3.2 \times 10^{-9}-1.0 \times 10^{-3} \mathrm{M}$ for MBA4/PPFP-Br) ATP concentrations. LOD determinations for each MBA/PPFP-Br combination were done in a similar way to that in Fig. 1b using four independent measurements for each MBA and the results are shown in Fig. 6b-e. From these plots one can determine the LODs to be $5.7 \times 10^{-4} \mathrm{M}$ for MBA1, $1.2 \times$ $10^{-5} \mathrm{M}$ for MBA2, $3.1 \times 10^{-7} \mathrm{M}$ for MBA3 and $4.6 \times 10^{-8} \mathrm{M}$ for MBA4. It is worth noting that in the case of MBA5, we measured a similar trend but the signal to noise ratio was insufficient due to the weak FRET signal (see Fig. S1 in the ESI†).

The trends observed for the different MBAs may be rationalized by focusing on the two extreme cases, namely MBA1 and MBA4. Looking at the conditions in Fig. 6a with $[\mathrm{ATP}]=0 \mathrm{M}$ allows one to probe the situation where the difference in FRET signal is primarily determined by the ability of PPFP-Br to disrupt the hairpin structure (see blue box in Scheme 2). Under these conditions, the larger FRET ratio observed with MBA1 vs. MBA4 ultimately reflects the easier opening of the weaker stem. In the $10^{-9}$ to $10^{-4} \mathrm{M}$ [ATP] range, there is no change in the FRET ratio with MBA1. Thus, the majority of species 3 is
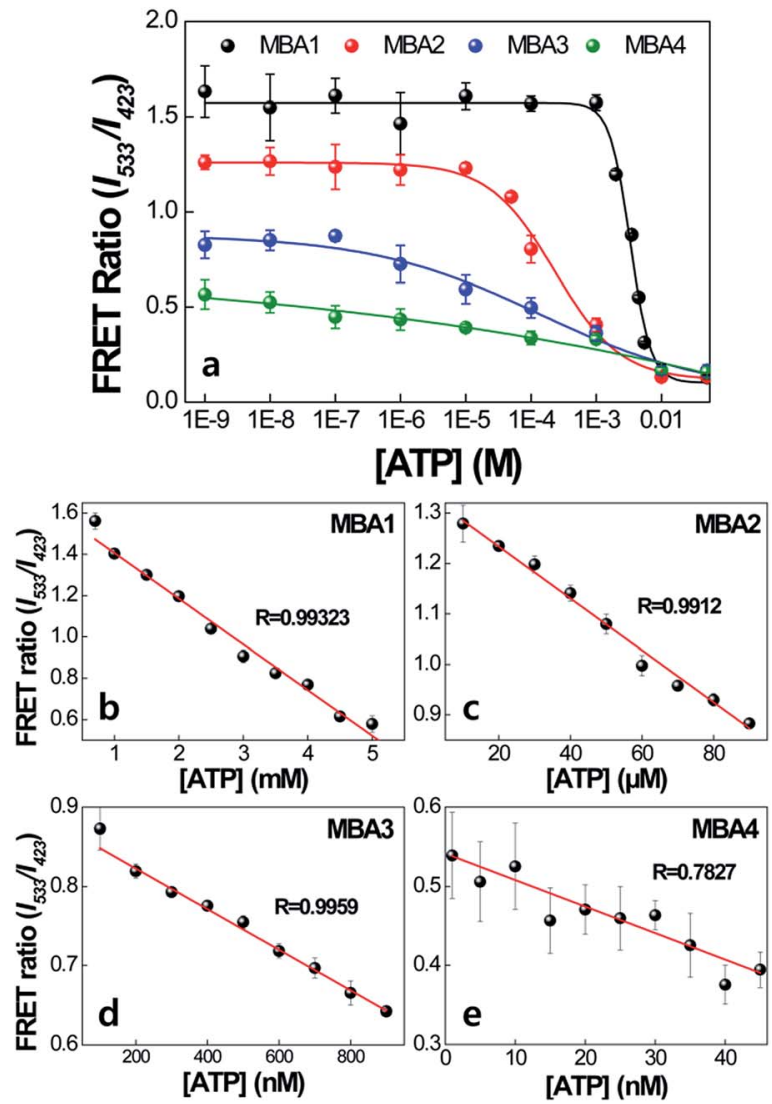

Fig. 6 Detection range of MBA/PPFP-Br assays as a function of [ATP]. $\left[\right.$ PPFP-Br] $=1.0 \times 10^{-6} \mathrm{M},[\mathrm{MBA}]=2.0 \times 10^{-8} \mathrm{M}$. The data were obtained from four independent measurements and the error bars indicate the standard deviation.

converted into $1^{\prime \prime}$ upon complexation with PPFP-Br. That the FRET ratio does change with MBA4 in this concentration range indicates a more resistant version of species 3 with the more stable stem structure. In other words, $K_{4}$ is larger for MBA1 than MBA4. At the high concentration regime, as one reaches the millimolar range, even with the weakest stem (namely MBA1), one can shift the equilibrium to species 3 in which 6-FAM emission is effectively quenched within the G-quadruplex structure. MBA2 and MBA3 provide intermediate examples of the stability continuum and create the opportunity to examine intermediate concentration ranges. Thus, the differences in concentration sensitivity profiles most reasonably trace their origins to the ability of PPFP-Br to disturb species 3, the stability of which is reasonably determined to a large extent by $K_{1}$. It is noted that as $K_{1}$ increases, $K_{3}$ and $K_{4}$ decrease, influencing the [ATP] range showing spectral responses.

The conformational change of MBAs with/without ATP and PPFP-Br was also studied by measuring the circular dichroism (CD) spectra (Fig. S2 $\dagger$ ). The characteristic absorption bands of the quadruplex (ATP/MBA1) at 265 and $295 \mathrm{~nm}$ were observed in the presence of $\operatorname{ATP}\left([\mathrm{MBA} 1]=1.0 \times 10^{-6} \mathrm{M},[\mathrm{ATP}]=5.0 \times 10^{-2}\right.$ M). Upon addition of PPFP-Br ([PPFP-Br $\left.]=5.0 \times 10^{-5} \mathrm{M}\right)$, the peak intensity of the quadruplex (ATP/MBA1) decreased, indicating a partial breakup of the G-quadruplex structure by 
PPFP-Br (structural transformation into the open-chain form). The CD data also support the weak binding affinity between ATP and the aptamer. However, almost identical CD spectra were obtained for ATP/MBA3 in the presence and absence of PPFP-Br, suggesting the tighter folded G-quadruplex formation relative to ATP/MBA1. This emphasizes the role of stem strength to compensate the weak binding affinity between the aptamer and ATP for fine-tuning the sensor characteristics.

The selectivity data of the MBA- and MBA/CPE-based ATP assays were measured against three ATP analogues, guanosine$5^{\prime}$-triphosphate (GTP), cytidine-5'-triphosphate (CTP) and uridine- $5^{\prime}$-triphosphate (UTP). The PL signals of MBA1-5 $(2.0 \times$ $10^{-8} \mathrm{M}$ ) were measured in the presence of GTP, CTP and UTP (5 $\mathrm{mM}$ ) by exciting 6-FAM directly at $490 \mathrm{~nm}$ without PPFP-Br. Because the ATP-specific aptamer recognizes the nucleoside unit of ATP, the binding affinity for other molecules is expected to be much weaker than ATP. The intensity ratio $I / I_{0}$ (where $I$ is the 6-FAM PL intensity at $517 \mathrm{~nm}$ and $I_{0}$ is the original intensity without analytes) was compared with that in the presence of ATP, where the most serious PL quenching was measured with ATP for all MBA probes (Fig. S4 $\dagger$ ), suggesting remarkable selectivity against ATP analogues for all MBA-based assays. With regard to the MBA/CPE-based assays, the FRET spectra of MBA1-4/PPFP-Br were measured in the presence of ATP analogues ( $5 \mathrm{mM}$ ). In Fig. 7, the FRET ratio was compared for all MBA/PPFP-Br bioassays in the presence of ATP and ATP analogues. Although a substantial decrease in the FRET signal was observed with CTP, the smallest FRET ratio was measured in the presence of ATP, suggesting a clear selectivity for ATP. With increasing the stem stability in MBAs, the FRET signal (on state without analytes) decreases with decreased on/off ratio, resulting in the deteriorated selectivity. The MBAs with the higher stem strength enhanced the detection sensitivity with the extended dynamic range with the sacrificed signal on/off ratio, where a clear trade-off relationship was observed.

We also measured the FRET PL spectra of MBA1/PPFP-Br in $20 \mathrm{mM}$ Tris-HCl buffer ( $\mathrm{pH}$ 7.4) with changing salts. The ions such as $\mathrm{K}^{+}, \mathrm{Mg}^{2+}$ and $\mathrm{Ca}^{2+}$ can influence the G-quadruplex

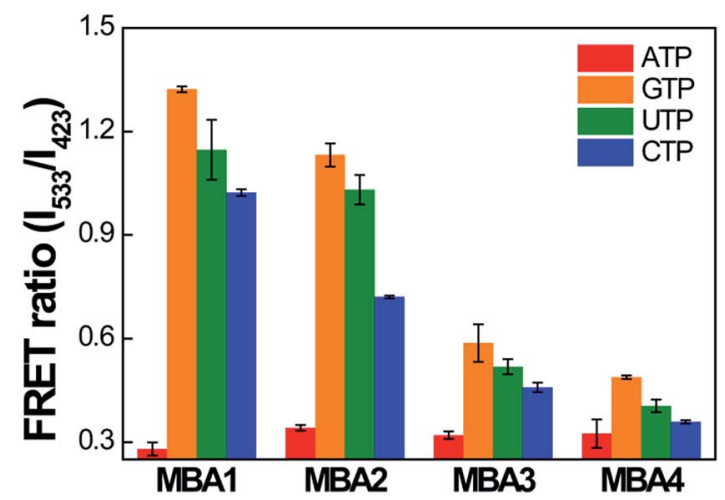

Fig. 7 Selectivity data for MBA/PPFP-Br assays. FRET ratio $\left(/ 533 / I_{423}\right)$ was compared in the presence of ATP and its analogues. $[\mathrm{MBA}]=2.0 \times$ $10^{-8} \mathrm{M}$, [PPFP-Br] $=1.0 \times 10^{-6} \mathrm{M}$, [ATP analogues] $=5 \mathrm{mM}$. The data were obtained from four independent measurements and the error bars indicate the standard deviation. folding/unfolding and/or MBA's conformational change. Fig. S5 $\uparrow$ shows the FRET signal in the presence of $100 \mathrm{mM} \mathrm{KCl}$, $\mathrm{MgCl}_{2}, \mathrm{CaCl}_{2}$ and all ions together. When compared to the case with $\mathrm{NaCl}$, the FRET ratio (without ATP) slightly decreases in the presence of these ions, indicting the hairpin structure is more stabilized with less efficient opening by CPEs. However, the FRET signal is clearly quenched upon addition of ATP for all cases, suggesting that the MBA/CPE sensory system can respond to the presence of ATP, even in the presence of all $\mathrm{K}^{+}, \mathrm{Mg}^{2+}$ and $\mathrm{Ca}^{2+}$ ions together.

\section{Conclusions}

In summary, we report how to combine synthetic CPEs and MBAs to create biosensor platforms that can be tuned to cover concentration ranges that extend beyond those determined by the specific aptamer-target binding constant. We also studied in detail the principal factors which govern the detection range tuning mechanism. In our specific example, the cationic PPFP$\mathrm{Br}$ was combined with a range of MBA structures that maintain the same ATP recognition sequence but are modified by the stem composition so to exhibit different structural stabilities. In this MBA/CPE assay (Scheme 2), $K_{2}$ is assumed to be nearly invariant because all MBAs have the same aptamer sequence in the loop, indicating no change in ATP binding affinity. The resulting FRET-sensitized signal depends on the differential opening of folded ATP/MBA G-quadruplexes by PPFP-Br, where the both ATP/aptamer binding $\left(K_{2}\right)$ and stem interactions $\left(K_{1}\right)$ compete with the MBA/PPFP-Br interaction. The equilibrium constant $K_{4}$ decreases with increasing $K_{1}$. With increasing stem stability, the folded ATP/MBA quadruplex structure becomes resistant to opening by PPFP-Br, even when [ATP] is low, which improves the limit of detection and broadens detection range.

From a practical perspective, the limit of detection was successfully modulated from millimolar (MBA1) to micromolar (MBA2), and further extended to nanomolar concentrations of ATP (MBA3 and MBA4). We note that [ATP] in living systems varies depending on the organelle and environment, typically 1$10 \mathrm{mM}$ in cells and $\sim 1 \mu \mathrm{M}$ in blood. The lower concentration level $(\sim \mathrm{nM})$ of human plasma ATP has also been reported. ${ }^{\mathbf{4 9 , 5 0}}$ To cover the wide range of target concentrations found in a human body, a range-tunable detection strategy such as that provided by the combination of plots in Fig. 6 would be highly desirable. ${ }^{22,51}$ This strategy has the potential to be extended to other chemical- and biological-assays with low target binding affinity and is likely to be made functional by a range of other cationic CPE structures.

\section{Experimental}

\section{General considerations}

All chemicals were purchased from Aldrich Chemical Co. and used as received unless otherwise mentioned. High pressure liquid chromatography (HPLC)-purified molecular beacon aptamers labeled with 6-FAM and DABCYL with 30-38 bases (MBA1: 5'-6-FAM-TACA CTGG GGAG TATT GCGG AGGA AGTG TA-DABCYL-3', MBA2: 5'-6-FAM-TGCG CTGG GGAG TATT 
GCGG AGGA AGCG CA-DABCYL-3', MBA3: 5'-6-FAM-GCGC GCGG GGAG TATT GCGG AGGA GCGC GC-DABCYL-3', MBA4: 5'-6-FAM-GCGC GCGC GGGG AGTA TTGC GGAG GAGC GCGC GC-DABCYL-3', MBA5: 5'-6-FAM-GCGC GCGC GCGG GGAG TATT GCGG AGGA GCGC GCGC GC-DABCYL-3') were obtained from Sigma-Genosys.

UV/vis absorption spectra were measured by using a Jasco (V630) spectrophotometer. The photoluminescence spectra were obtained on a Jasco (FP-6500) spectrofluorometer with a Xenon lamp excitation source, using $90^{\circ}$ angle detection for the solution samples. The fluorescence quantum yield was measured relative to a standard fluorescein solution in water at $\mathrm{pH}=11$. The circular dichroism spectra were obtained using a Jasco (J715) 202 circular dichroism spectrometer.

\section{Synthesis and characterization of CPE (PPFP-Br)}

The cationic CPE, poly[( $\left(9,9^{\prime}\right.$-bis(4-(6-N,N,N-trimethylammoniumhexyloxy)phenyl)fluorene-2,7-diyl)-alt-1,4-phenylene dibromide] (PPFP-Br) was synthesized following previously reported procedures. ${ }^{52,53}$ The number average molecular weight of PPFP-Br was determined to be $\mathrm{Mn}=16000 \mathrm{~g} \mathrm{~mol}^{-1}$ (polydispersity index $=2.7$ ) by examination of the neutral precursor by gel permeation chromatography relative to polystyrene standards. The absorption and PL maxima of PPFP-Br were measured at $\lambda_{\mathrm{abs}}=375 \mathrm{~nm}$ and $\lambda_{\mathrm{PL}}=423 \mathrm{~nm}$, respectively, in a $20 \mathrm{mM}$ Tris-HCl buffer solution with $100 \mathrm{mM} \mathrm{NaCl}$. PPFP-Br shows a PL quantum efficiency of $\sim 46 \%$ in this buffer. The emission of PPFP-Br shows a good spectral overlap with the absorption of 6-FAM $\left(\lambda_{\text {abs }}=490 \mathrm{~nm}\right)$ as the FRET acceptor (Fig. S3 in the ESI†).

\section{ATP detection assay protocols}

PL experiments were performed in $20 \mathrm{mM}$ Tris- $\mathrm{HCl}$ buffer $(\mathrm{pH}=$ 7.4) containing $100 \mathrm{mM} \mathrm{NaCl}$. A stock solution $\left(10^{-5} \mathrm{M}\right)$ of each MBA was prepared in deionized water. Each MBA stock solution $(4 \mu \mathrm{L})$ was added to $2 \mathrm{~mL}$ buffer and the resulting solutions were incubated around the melting temperatures of MBAs (at $65{ }^{\circ} \mathrm{C}$ for MBA1, $75{ }^{\circ} \mathrm{C}$ for MBA2, $80^{\circ} \mathrm{C}$ for MBA3 and $90^{\circ} \mathrm{C}$ for MBA4 and 5 , respectively) for $20 \mathrm{~min}$ with changing [ATP]. The annealed solution was cooled down slowly to room temperature for $1.5 \mathrm{~h}$ PPFP-Br (20 $\mu \mathrm{L}, 10^{-4} \mathrm{M}$ in water/dimethylsulfoxide (3 vol\%)) was then added to the above solution and the PL spectra were measured by exciting either the polymer or 6-FAM. The final concentration of MBA and PPFP-Br in the assay system was $2.0 \times 10^{-8} \mathrm{M}$ and $1.0 \times 10^{-6} \mathrm{M}$, respectively. A charge ratio $([+] /[-],[+]$ in PPFP-Br: $[-]$ in MBA) of $\sim 3$ was chosen for measuring PL spectra.

For the opening test of MBAs by PPFP-Br, the MBA solution $\left([\mathrm{MBA}]=2.0 \times 10^{-8} \mathrm{M}\right)$ in Tris-HCl buffer was annealed around its melting temperature for $20 \mathrm{~min}$ and cooled down to room temperature over a period of $1.5 \mathrm{~h}$ to achieve the equilibrium state. The PL spectra were measured by exciting 6-FAM at 490 $\mathrm{nm}$ with increasing [PPFP-Br] $=0-1.0 \times 10^{-6} \mathrm{M}$ at room temperature by successive addition of $4 \mu \mathrm{L}$ of aq. stock solution $\left(10^{-4} \mathrm{M}\right)$ of PPFP-Br.

\section{Acknowledgements}

We are grateful for financial support from the National Research Foundation (NRF) of Korea (2012R1A2A2A06045327, 2013M3C1A3065522, 2012M3A6A7055540) and from the Institute for Collaborative Biotechnologies (ICB) under grant W911F09-D-0001 from the U.S. Army Research Office.

\section{Notes and references}

1 A. Herland and O. Inganäs, Macromol. Rapid Commun., 2007, 28, 11.

2 S. W. Thomas III, G. D. Joly and T. M. Swager, Chem. Rev., 2007, 107, 48.

3 J. Liang, K. Li and B. Liu, Chem. Sci., 2013, 4, 18.

4 A. Duarte, K.-Y. Pu, B. Liu and G. C. Bazan, Chem. Mater., 2011, 23, 15.

5 H. Jiang, P. Taranekar, J. R. Reynolds and K. S. Schanze, Angew. Chem., Int. Ed., 2009, 48, 17.

6 F. Feng, L. Liu and S. Wang, Nat. Protoc., 2010, 5, 10.

7 D. T. McQuade, A. E. Pullen and T. M. Swager, Chem. Rev., 2000, 100, 38.

8 B. S. Gaylord, M. R. Massie, S. C. Feinstein and G. C. Bazan, Proc. Natl. Acad. Sci. U. S. A., 2005, 102, 6.

9 B. S. Gaylord, A. J. Heeger and G. C. Bazan, Proc. Natl. Acad. Sci. U. S. A., 2002, 99, 4.

10 K. Lee, L. K. Povlich and J. Kim, Adv. Funct. Mater., 2007, 17, 8.

11 C. A. Traina, R. C. Bakus II and G. C. Bazan, J. Am. Chem. Soc., 2011, 133, 8.

12 K. Lee, J. Lee, E. J. Jeong, A. Kronk, K. S. J. Elenitoba-Johnson, M. S. Lim and J. Kim, Adv. Mater., 2012, 24, 2479.

13 F. Wang, Z. Liu, B. Wang, L. Feng, L. Liu, F. Lv, Y. Wang and S. Wang, Angew. Chem., Int. Ed., 2013, 52, 1.

14 X. Feng, F. Lv, L. Liu, Q. Yang, S. Wang and G. C. Bazan, Adv. Mater., 2012, 24, 5428.

15 Y. C. Shin, J. H. Lee, J.-E. Jeong, B. Kim, E. J. Lee, O. S. Jin, T. G. Jung, J. J. Lee, H. Y. Woo and D.-W. Han, Biotechnol. Appl. Biochem., 2013, 60, 580.

16 V. S. Le, B. Kim, W. Lee, J.-E. Jeong, R. Yang and H. Y. Woo, Macromol. Rapid Commun., 2013, 34, 772.

17 B. L. Nguyen, J.-E. Jeong, I. H. Jung, B. Kim, V. S. Le, I. Kim, K. Kyhm and H. Y. Woo, Adv. Funct. Mater., 2014, 24, 1748. 18 Q. Zhou and T. M. Swager, J. Am. Chem. Soc., 1995, 117, 2. 19 T. M. Swager, Acc. Chem. Res., 1998, 31, 201.

20 A. Tsourkas, M. A. Behlke, S. D. Rose and G. Bao, Nucleic Acids Res., 2003, 31, 1319.

21 A. Vallée-Bélisle, F. Ricci and K. W. Plaxco, Proc. Natl. Acad. Sci. U. S. A., 2009, 106, 13802.

22 A. Vallée-Bélisle, F. Ricci and K. W. Plaxco, J. Am. Chem. Soc., 2012, 134, 2876.

23 D. Kang, A. Vallée-Bélisle, A. Porchetta, K. W. Plaxco and F. Rici, Angew. Chem., Int. Ed., 2012, 51, 6717.

24 B. Kim, I. H. Jung, M. Kang, H.-K. Shim and H. Y. Woo, J. Am. Chem. Soc., 2012, 134, 3133.

25 F. He, Y. Tang, M. Yu, F. Feng, L. An, H. Sun, S. Wang, Y. Li, D. Zhu and G. C. Bazan, J. Am. Chem. Soc., 2006, 128, 6764. 
26 Y. Y. Wang and B. Liu, Langmuir, 2009, 25, 12787.

27 B. Li, C. Qin, T. Li, L. Wang and S. Dong, Anal. Chem., 2009, 81,3544 .

28 T. Hermann and D. J. Patel, Science, 2000, 287, 820.

29 A. D. Ellington and J. W. Szostak, Nature, 1990, 346, 818.

30 J. T. Davis, Angew. Chem., Int. Ed., 2004, 43, 668.

31 V. Esposito, A. Galeone, L. Mayol, G. Oliviero, A. Virgilio and L. Randazzo, Nucleosides, Nucleotides Nucleic Acids, 2007, 1155.

32 H. Yaku, T. Fujimoto, T. Murashima, D. Miyoshi and N. Sugimoto, Chem. Commun., 2012, 48, 6203.

33 S. Tyagi and F. R. Kramer, Nat. Biotechnol., 1996, 14, 303.

34 F. Xia, X. Zuo, R. Yang, Y. Xiao, D. Kang, A. Vallée-Bélisle, X. Gong, A. J. Heeger and K. W. Plaxco, J. Am. Chem. Soc., 2010, 132, 1252.

35 D. E. Huizenga and J. W. Szostak, Biochemistry, 1995, 34, 656. 36 Y. Wang, Z. Li, D. Hu, C.-T. Lin, J. Li and Y. Lin, J. Am. Chem. Soc., 2010, 132, 9274.

37 C.-L. Zhu, C.-H. Lu, X.-Y. Song, H.-H. Yang and X.-R. Wang, J. Am. Chem. Soc., 2011, 133, 1278.

38 J. Liu and Y. Lu, Angew. Chem., Int. Ed., 2006, 45, 90.

39 X. He, Y. Zhao, D. He, K. Wang, F. Xu and J. Tang, Langmuir, 2012, 28, 12909.
40 J. J. Li, Z. Fang and W. Tan, Biochem. Biophys. Res. Commun., 2002, 292, 31.

41 V. Bagalkot, O. C. Farokhzad, R. Langer and S. Jon, Angew. Chem., Int. Ed., 2006, 45, 1.

42 J.-H. Lee, M. D. Canny, A. D. Erkenez, D. Krilleke, Y.-S. Ng, D. T. Shima, A. Pardi and F. Jucker, Proc. Natl. Acad. Sci. U. S. A., 2005, 102, 18902.

43 J. C. Cox and A. D. Ellington, Bioorg. Med. Chem., 2001, 9, 2525.

44 C. H. Lin and D. J. Patel, Chem. Biol., 1997, 4, 817.

45 A. V. Hill, J. Physiol., 1910, 40, IV.

46 A. Porchetta, A. Vallée-Bélisle, K. W. Plaxco and F. Ricci, J. Am. Chem. Soc., 2012, 134, 20601.

47 The UNAFold Web Server, http://mfold.rna.albany.edu, accessed 1995.

48 M. Zuker, Nucleic Acids Res., 2003, 31, 3406.

49 I. Beis and E. A. Newsholme, Biochem. J., 1975, 152, 23.

50 M. W. Gorman, E. O. Feigl and C. W. Buffington, Clin. Chem., 2007, 53, 318.

51 F. Ricci, A. Vallée-Bélisle, A. Porchetta and K. W. Plaxco, J. Am. Chem. Soc., 2012, 134, 15177.

52 J.-H. Lee and D.-H. Hwang, Chem. Commun., 2003, 2836.

53 B. H. Lee, I. H. Jung, H. Y. Woo, H.-K. Shim, G. Kim and K. Lee, Adv. Funct. Mater., 2014, 24, 1100. 